\title{
Structure of Halothiobacillus neapolitanus Carboxysomes by Cryo-electron Tomography
}

\author{
Michael F. Schmid ${ }^{1 *}$, Angel M. Paredes ${ }^{1}$, Htet A. Khant ${ }^{1}$, Ferda Soyer ${ }^{2}$ \\ Henry C. Aldrich ${ }^{*}$, Wah Chiu ${ }^{1}$ and Jessup M. Shively ${ }^{3}$
}

\author{
${ }^{1}$ National Center for \\ Macromolecular Imaging \\ Verna and Marrs McLean \\ Department of Biochemistry \\ and Molecular Biology \\ Baylor College of Medicine \\ Houston, TX 77030, USA \\ ${ }^{2}$ Department of Biology \\ Izmir Institute of Technology \\ Izmir, Turkey \\ ${ }^{3}$ Department of Genetics and \\ Biochemistry, Clemson \\ University, Clemson \\ SC 29634, USA
}

\begin{abstract}
Carboxysomes are polyhedral bodies consisting of a proteinaceous shell filled with ribulose 1,5-bisphosphate carboxylase/oxygenase (RuBisCO). They are found in the cytoplasm of all cyanobacteria and some chemoautotrophic bacteria. Previous studies of Halothiobacillus neapolitanus and Nitrobacter agilis carboxysomes suggest that the structures are either icosahedral or dodecahedral. To determine the protein shell structure more definitively, purified $H$. neapolitanus carboxysomes were re-examined by cryo-electron tomography and scanning transmission electron microscopy (STEM). Due to the limited tilt angles in the electron microscope, the tomographic reconstructions are distorted. Corrections were made in the 3D orientation searching and averaging of the computationally extracted carboxysomes to minimize the missing data effects. It was found that $H$. neapolitanus carboxysomes vary widely in size and mass as shown by cryoelectron tomography and STEM mass measurements, respectively. We have aligned and averaged carboxysomes in several size classes from the 3D tomographic reconstruction by methods that are not model-biased. The averages reveal icosahedral symmetry of the shell, but not of the density inside it, for all the size classes.
\end{abstract}

(C) 2006 Elsevier Ltd. All rights reserved. tron tomography

\section{Introduction}

The initial source of Earth's organic carbon is atmospheric or dissolved $\mathrm{CO}_{2}$, which is fixed by enzymes found in green plants, algae and autotrophic bacteria. The carbon is then spread through the food chain to all other living organisms. The most important enzyme responsible for fixing carbon is ribulose bisphosphate carboxylase/oxygenase (RuBisCO), which catalyzes the first step in carbon fixation via the Calvin-Benson-Bassham cycle, the covalent attachment of $\mathrm{CO}_{2}$ to ribulose-1,5-bisphosphate and its subsequent cleavage into two molecules of 3-phosphoglycerate. Based on the immense

\section{Deceased.}

Present address: A. M. Paredes, University of Texas Houston Health Science Center, Houston, TX 77030, USA.

Abbreviations used: RuBisCO, ribulose 1,5bisphosphate carboxylase/oxygenase; STEM, scanning transmission electron microscopy; FSC, Fourier shell coefficient. biomass of photosynthetic and chemoautotrophic organisms on Earth, RuBisCO is estimated to be the most abundant protein known.

All cyanobacteria and some chemoautotrophic bacteria enhance their $\mathrm{CO}_{2}$ fixation by sequestering RuBisCO into polyhedral bodies called carboxysomes. ${ }^{1-4}$ Halothiobacillus neapolitanus carboxysomes are delimited by a proteinaceous shell and are filled with RuBisCO. ${ }^{5-7}$ In previous electron microscopic studies, carboxysomes appeared hexagonal with a granular interior, a diameter of approximately $120 \mathrm{~nm}$ and a shell thickness of between $3 \mathrm{~nm}$ and $4 \mathrm{~nm} .8,9$ Although the enhancement of carbon dioxide fixation by the carboxysome has been firmly established, the exact mechanism has not yet been elucidated.

$H$. neapolitanus carboxysomes consist of ten polypeptides. ${ }^{6,7}$ Of these, two polypeptides represent the small and large subunits of RuBisCO and six are known to make up the shell. One of these shell peptides has been identified as a unique carbonic anhydrase. ${ }^{10}$ It is postulated that this enzyme may function to enhance carbon dioxide fixation by the carboxysome. The functions and locations of the 
two remaining polypeptides are not yet known. Carboxysomes are rapidly formed via de novo mRNA and protein synthesis under low $\mathrm{CO}_{2}$ conditions. ${ }^{11,12}$ Their genes are operon-linked, ${ }^{12}$ so it is likely the proportions of their constituents are regulated.

Although much has been published on the occurrence, physiology, biochemistry and genetics of these microcompartments/organelles, ${ }^{5-7,10}$ only two major reports have analyzed the structure or symmetry of purified carboxysomes. ${ }^{9,13}$ Peters concluded that the carboxysomes of Nitrobacter agilis obey icosahedral symmetry, based on negative stain projection images and heavy-metal shadowing of critical-point-dried carboxysomes. ${ }^{13}$ Holthuijzen and co-workers, on the other hand, reported the shape of carboxysomes of $H$. neapolitanus to be dodecahedral. ${ }^{9}$

There are reports that the $\mathrm{RuBisCO}$ inside the shell is paracrystalline and fills the interior. ${ }^{8}$ Others have reported that the $\mathrm{RuBisCO}$ molecules are arranged in one or a few layers under the surface of the shell. ${ }^{9}$ However, these conclusions were drawn from observations of either whole bacteria or isolated carboxysome preparations that are plastic-embedded, thinsectioned, and/or negative stained. Using Hilbert Differential Contrast electron microscopy, paracrystalline arrays were observed in intact frozen cells, ${ }^{14}$ but it is not possible in these 2D projections to tell whether the carboxysomes are filled, or have only one or a few layers of RuBisCO under the shell. Orus et al. ${ }^{11}$ reported that the state of packaging of RuBisCO inside carboxysomes can vary with environmental conditions.

It is these aspects of the carboxysome structure that we intend to clarify using cryo-electron tomography of suspensions of carboxysomes isolated from the chemoautotrophic proteobacterium $H$. neapolitanus and subsequent 3D alignment and averaging of particles computationally isolated from the tomogram. Averaging particles is necessary because of the low signal-to-noise ratio in any single instance of a particle from the tomogram, and because of the distortion introduced by the "missing wedge" of data in Fourier space. However, the search for the relative orientation of the $3 \mathrm{D}$ particle volumes is not a trivial step unless a 3D model of the particle with isotropic resolution already exists, as has been done. ${ }^{15,16}$ In our study, an orientation search method that does not depend on such a prior model is used for aligning single particles from a tomogram with missing wedge data.

\section{Results}

\section{Cryo-electron tomographic Tilt series images}

Figure 1 shows the zero tilt image from a tilt series of a frozen-hydrated suspension of isolated carboxysomes. All the images in the tilt series are shown in Supplementary Data, Movie 1. The thin, polyhedral

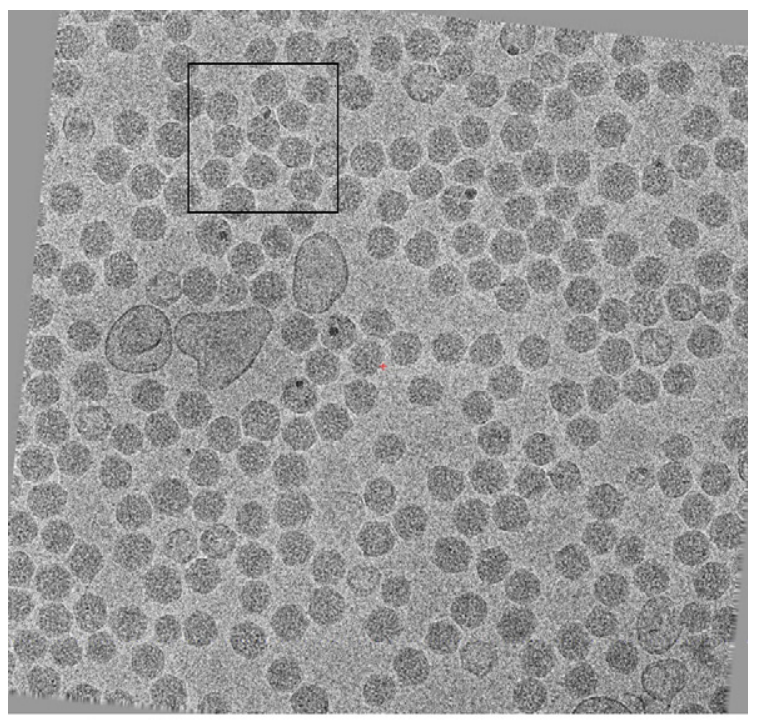

Original tilt series $0^{\circ}$ tilt image

Figure 1. An image from the tilt series near the $0^{\circ}$ tilt angle showing isolated carboxysomes embedded in a layer of vitreous ice. The entire series is represented in a movie as Supplementary Data, Movie 1. The box outlines the approximate area of the tomogram shown in Figure 2(a) and (b), about 400 pixels, or $432 \mathrm{~nm}$ on a side.

nature of the shell of the carboxysomes and their globular contents, presumed to include RuBisCO, are apparent. Also evident is that the particles appear to possess marked size variability.

\section{Tomographic reconstruction}

Figure 2(a) is a view of a representative area of the tomogram. Four consecutive sections from the middle of the tomogram are averaged along $z$. Features annotated in this view are the shell, which is about $4 \mathrm{~nm}$ thick, the slightly thicker and denser vertices, the RuBisCO molecules inside the particles, and that some particles are more fully packed than others. Figure 2(b) is a stereo view of the same area of the tomographic reconstruction. The entire tomogram is shown in Supplementary Data, Movie 2. From this raw tomogram we were able to gather insight into this preparation of carboxysomes. As mentioned, we observed that a few of the carboxysome shells were completely empty. In several of them, only part of the interior was occupied. In these particles, the preferred arrangement of RuBisCO was sometimes seen as a layer or two apposed to the inside surface of the shell. However, the majority of the particles had what appears to be a homogeneous distribution of RuBisCO throughout the interior of the shell. Inspection of this entire reconstruction from a suspension of isolated carboxysomes (Supplementary Data, Movie 2) showed that there were a number of small "free" particles outside the shells that appeared very similar to the interior RuBisCO molecules of carboxysomes. Their distribution was not random. They were preferentially disposed near 
Tomographic Reconstruction

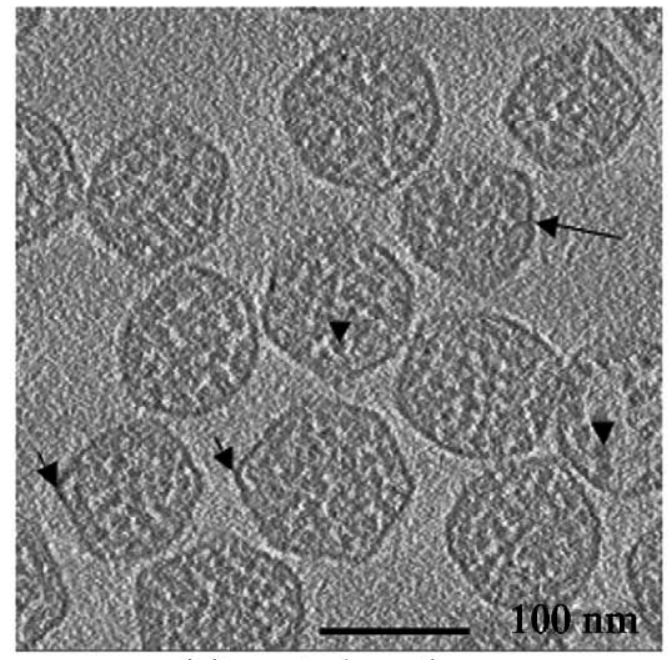

(a) central section

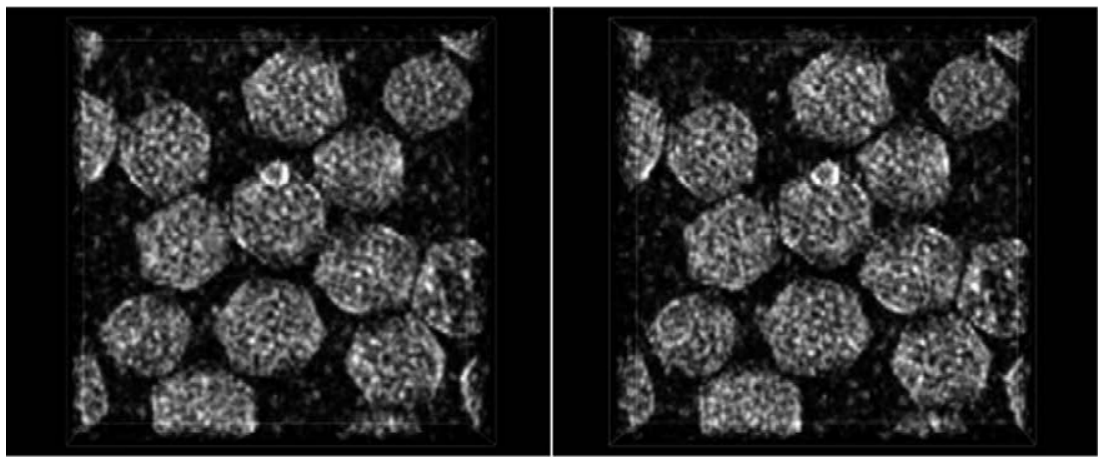

(b) stereo view
Figure 2. (a) A slice through the center of the tomographic volume in the area indicated in Figure 1. Four sections of the tomogram are averaged in $z$. A long arrow points to the thin shell, about $4 \mathrm{~nm}$ thick. Shorter arrows indicate the 5 -fold vertices of several particles where the shell is slightly thicker. Arrowheads indicate $\mathrm{RuBisCO}$ molecules inside the carboxysome shell. Some shells are fully packed others (on the right, for instance) are rather emptier. (b) Stereo image of a part of the tomogram, averaged by a factor of 4 $(4.32 \mathrm{~nm} /$ pixel) from the original image sampling and volume-rendered. A variety of sizes and orientations of carboxysomes are visible, and the amount of RuBisCO in the interior of these carboxysomes is also seen to vary (note the carboxysome near the lower right which has less mass inside). The majority of the free particles outside the carboxysomes in this preparation are likely to be RuBisCO molecules that have been liberated from the particles during the preparation. They preferentially congregate at the top and bottom of the vitreous ice. "Below" the particle near the center is some ice contamination on the "bottom" surface of the vitreous ice layer. The field of view spans $432 \mathrm{~nm}$ in width. The entire tomogram is shown as a movie in Supplementary Data, Movie 2. the top and bottom of the reconstruction and appeared to be attracted to the air-water interface (Supplementary Data, Movie 2). In addition, there are large particles at both surfaces of the vitreous ice that appeared to be ice contamination. The carboxysomes appear to lie between these two interfaces and to be well embedded in the vitreous ice as evidenced from the tomogram. From these observations and our assumption about the ice contamination and the "free" particles indicating the limits of the ice layer, we estimate the ice to be about 100$150 \mathrm{~nm}$ thick, slightly larger than the diameter of the carboxysomes themselves.

\section{Single particle orientation searching: dealing with the missing wedge}

There were about 200 carboxysomes visible in this single 3D tomographic reconstruction. Of these, 160 were chosen that were not obviously malformed or broken, and were not too close to the edge of the field of view. Some carboxysomes that were near the edges did not appear in every tilt series image because of the shifting field of view between different tilts in the tilt series (see Supplementary Data, Movie 1). The 3D volumes containing these 160 particles were extracted from the tomogram and centered by autocorrelation with their corres- ponding mirror images. To align and merge these particles to each other in 3D in an unbiased way, an all-versus-all comparison in 3D was envisioned. However, this would have involved $160 \times 159 / 2$ searches of the six dimensional parameter space (three rotation and three translation), and was deemed unfeasible. Therefore the data were split up into eight random groups of 20 particles each, and all-versus-all $3 \mathrm{D}$ orientation searches, followed by averaging in 3D, when appropriate, were done for each group.

We encountered a difficulty in these comparisons having to do with the geometry of data collection for tomographic reconstructions. Because a tilt angle range of $\pm 90^{\circ}$ cannot be achieved in the electron microscope, central sections in Fourier space for tilts higher than $60-70^{\circ}$ are missing, leading to the problem commonly referred to as the "missing wedge" in Fourier space. After extraction from the tomogram and before applying any rotations, the orientation of the missing wedge in Fourier space is identical for each particle/volume. However, the orientations of the particles themselves are random with respect to each other and must be searched for in $3 \mathrm{D}$ rotation space. Because of the strong signal from the pattern of the missing wedge, the crosscorrelation map may merely lead to the alignment of the missing wedges, which is certainly not correct. This problem does not occur to the same extent in 
cases where an isotropic starting model is available and used for 3D alignment. ${ }^{15,16}$

As two 3D volumes in Fourier space, each containing a missing wedge are rotated against each other in the cross-correlation search, and one volume is multiplied by the complex conjugate of the other, zeros are generated. They occur when the missing-data region for one of the particles is multiplied by data in the other particle and vice versa, and the number of such zeros changes at each rotation angle increment in the search. To minimize the effect of the missing wedge on the overall scale of the 3D cross-correlation map, we computed the number of non-zero terms after the complex multiplication in Fourier space for each relative $3 \mathrm{D}$ orientation in the space searched for the two particles, and scaled the 3D cross-correlation map at that orientation search angle by the reciprocal of this number. This correction factor is approximately right because the total power in the Fourier transform of a cross-correlation function influences the values of the cross-correlation map. This normalization was good enough to allow the true proper relative orientation of the particles to be determined as demonstrated from simulated data with a missing wedge introduced (M.F. S., unpublished results).

In each of the eight groups in this data set, the six parameters which yielded the highest cross-correlation coefficients between pairs of particles were used to align and average these pairs in 3D, and then the averages were used as new search models. New particles were added to these averages, gradually including more particles. This iterative approach is the same as used for the images of acrosomal bundle for unbiased merging. ${ }^{17}$ Each of the eight groups yielded one to four classes of particles that merged well with each other in that class but not between each other in that group. The structural basis of this classification was not investigated at this stage. About half the particles in each group fell into these classes, yielding a total of 92 particles that seemed to be in self-consistent classes. The purpose of this initial classification was to generate subsets of particles that shared a similarity to at least a few others, and were not unique outliers.

\section{Particle averaging: classification of heterogeneous structures}

The second step of data classification was to divide the 92 particles up by size, because considerable size variation was still seen in the particles after the initial classification step. EMAN software was used to calculate the $1 \mathrm{D}$ radial density profiles from the 3D density map of each particle. They were classified according to the maximum radius of the 1D plot. Figure 3 is a histogram of the particle diameters based on these radii. The histogram was initially divided up arbitrarily into nine size classes, from $88 \mathrm{~nm}$ to $106 \mathrm{~nm}$ in average diameter. Again, allversus-all 6D cross-correlation searching and merging was done and the averages for each size class were created, containing up to 21 particles in each class. All the particles were cross-correlated against these averages. The particles usually correlated best with the size class that originally contained them, although a few correlated better with the size class above or below them, and were thus shifted into these classes. The result of this shifting was that the members of two of the original nine size classes migrated to other groups, and thus at the end, only seven stable size classes remained. Iterative merging was done with progressively finer steps of rotation search, down to one-degree intervals. This produced the final size-classified averages. For these final averages, we scaled the contributions of each particle by weighting each voxel's contribution in Fourier space by its fraction of the total amplitude. The major effect of this correction, as expected, was to increase the visibility of the thin shell where it is oriented perpendicular to the $z$ direction, while the other features were basically unchanged.

Figure 4(a) shows the average of the most highly populated class with no symmetry enforced, having a diameter of about $100 \mathrm{~nm}$. It appears to obey icosahedral symmetry as characterized by the appearance of 5 (approximately along the view direction), 3 and 2-fold symmetry axes and 20 triangular faces. Similar averages were produced for other size classes and also have similar symmetry appearance. This observation justifies the appli-

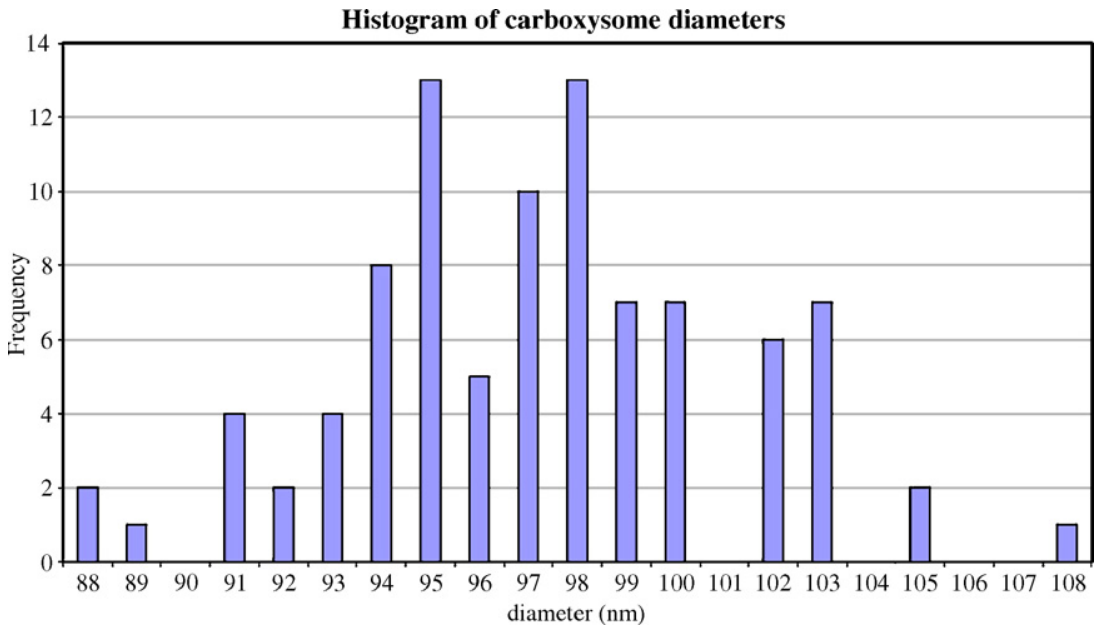

Figure 3. A histogram of the diameters of the carboxysomes in the data set. The diameters of the particles were determined from the 1D radial density profiles of each of the $923 \mathrm{D}$ extracted and centered volumes. Each bin is $1 \mathrm{~nm}$ wide, from $88 \mathrm{~nm}$ to $108 \mathrm{~nm}$. A large group of particles cluster around a size of 94-95 nm and 97-100 nm, but groups with larger and smaller size particles are clearly present. 
(a)

\section{Tomographic averaging - 100nm class}

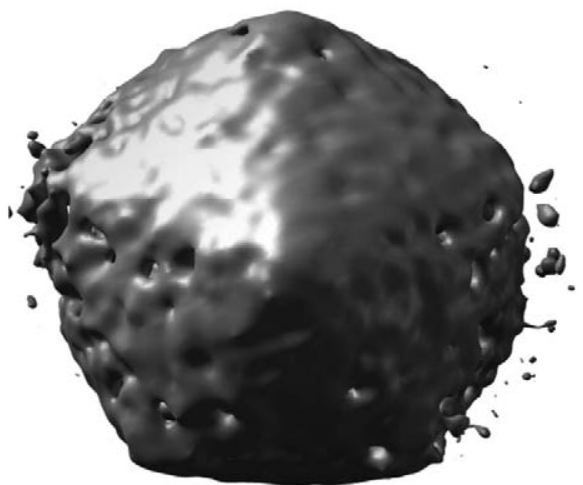

(b)

Tomographic plus icosahedral averaging - $100 \mathrm{~nm}$ class

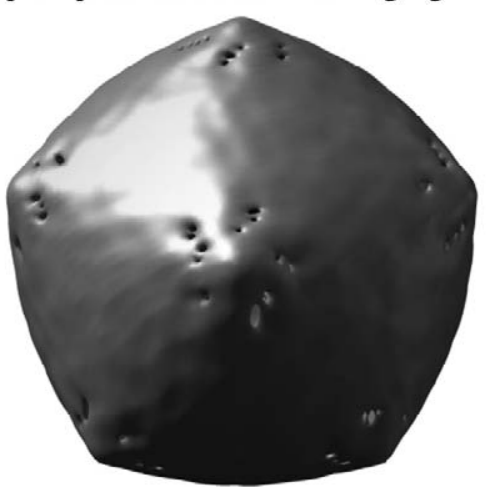

Figure 4. (a) The average of $213 \mathrm{D}$ reconstructions of particles averaging about $100 \mathrm{~nm}$ in diameter and surfacerendered. The average has no symmetry imposed, but it clearly displays approximate 5,3 and 2-fold symmetry in the correct relationships to produce icosahedral symmetry. The direction along which the resolution is affected by the missing wedge in Fourier space is approximately horizontal in this view. (b) The average in (a) was icosahedrally averaged. This average is also displayed in Figure 5(f) as part of the gallery of particle averages from all the size classes.

cation of icosahedral averaging as shown in Figure 4(b). Figure 5(a)-(g) shows a gallery of views of all seven surviving size class averages with icosahedral symmetry enforced. The Fourier shell coefficient (FSC) was calculated by splitting the 3D volumes for the largest class of particles, whose overall average is shown in Figure 4(b), into two separate averages (odd and even numbered members). The resolution as indicated by the 0.5 threshold of FSC is $6.6 \mathrm{~nm}$.

\section{The structure: shell}

The shell of the carboxysome is rather thin, about 3-4 nm thick (see Figure 2(a) and Supplementary Data, Movie 2). At the vertices, there is a small "button" of higher density in all the size classes (Figure 2(a)). One of the major differences between the seven different size classes of carboxysomes (Figure 5(a)-(g)) is the organization of the density at the center of the triangular faces. One type of organization, followed by the shells of average diameter 88, 93, 95 and $103 \mathrm{~nm}$, has a higher density at the center of the face, surrounded by three or six densities abutting it (arrow, Figure 5(a)). It is interesting that the same spacing is seen in the sheets of hexamers in the crystal structure of a homologous shell protein from the carboxysome of Synechocystis and whose hexagonal organization in the crystal lattice was proposed to be a model for the carboxysome shell. ${ }^{18}$ The other type of organization, found in shells of diameter 91, 97 and $100 \mathrm{~nm}$, has three densities surrounding the center of the 3-fold face instead of being at the center itself as above, again with spacing similar to those in the crystal (arrow, Figure 5(b)). In addition to this difference in the arrangement for different size classes, the orientations of the densities with respect to the vertices of the shell are different in the different size averages. This suggests that the organization of the shell proteins is different for different size classes.

In addition to the Csos1A and C polypeptides, which comprise $13 \%$ of the carboxysome's mass ${ }^{5}$ and are homologous to CcmK2 and K4 in the crystal structure, there are up to five other proteins present in the shell.

The crystal structure of the carbonic anhydrase of $H$. neapolitanus, ${ }^{19}$ which is thought to be a stoichiometrically minor component of the shell $(2 \%$ of the carboxysome's mass $\left.{ }^{2,5}\right)$, is too thick to be part of the triangular faces. One possible hypothesis is that it is located at the thicker 5-fold vertex, where there is sufficient volume for it in all dimensions. If so, there may be only 60 copies per carboxysome, the minimum required for icosahedral symmetry.

\section{The structure: interior densities}

Each individual carboxysome shows globular features inside the carboxysome having density comparable to the shell (e.g. Figure 2(a) and (b); Supplementary Data, Movie 2 movie; and Figure 6(a) showing a radial density plot of an individual particle). However, Figure 6(b) shows that in the multi-particle and icosahedrally averaged reconstructions, the density inside the shell is much lower on average than the density of the shell. This would indicate that, while the shell is definitely icosahedral, the interior density, which is likely to include RuBisCO molecules, is probably not. However, the inside density in the radial or icosahedral averages is still concentrated in layers approximately equally spaced from each other and starting at about $6 \mathrm{~nm}$ from the inside of the shell (Figure 6(a) and (b)). This first layer under the shell in the icosahedral averages always has the highest density. From this we deduce that the RuBisCO has a certain probability of being at these various radii, with the most probable location being under the shell, but also able to populate the entire volume of the carboxysome. The locations of the average densities indicate that the average number and arrangement of RuBisCO molecules per layer could be different in different size classes. 


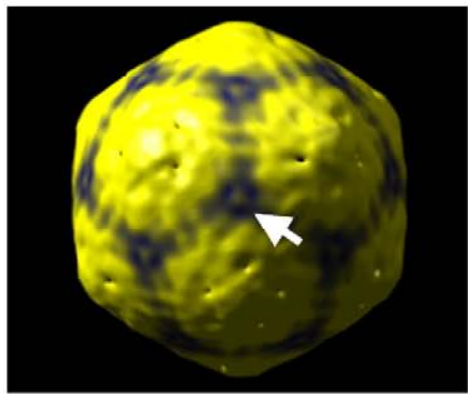

(a)
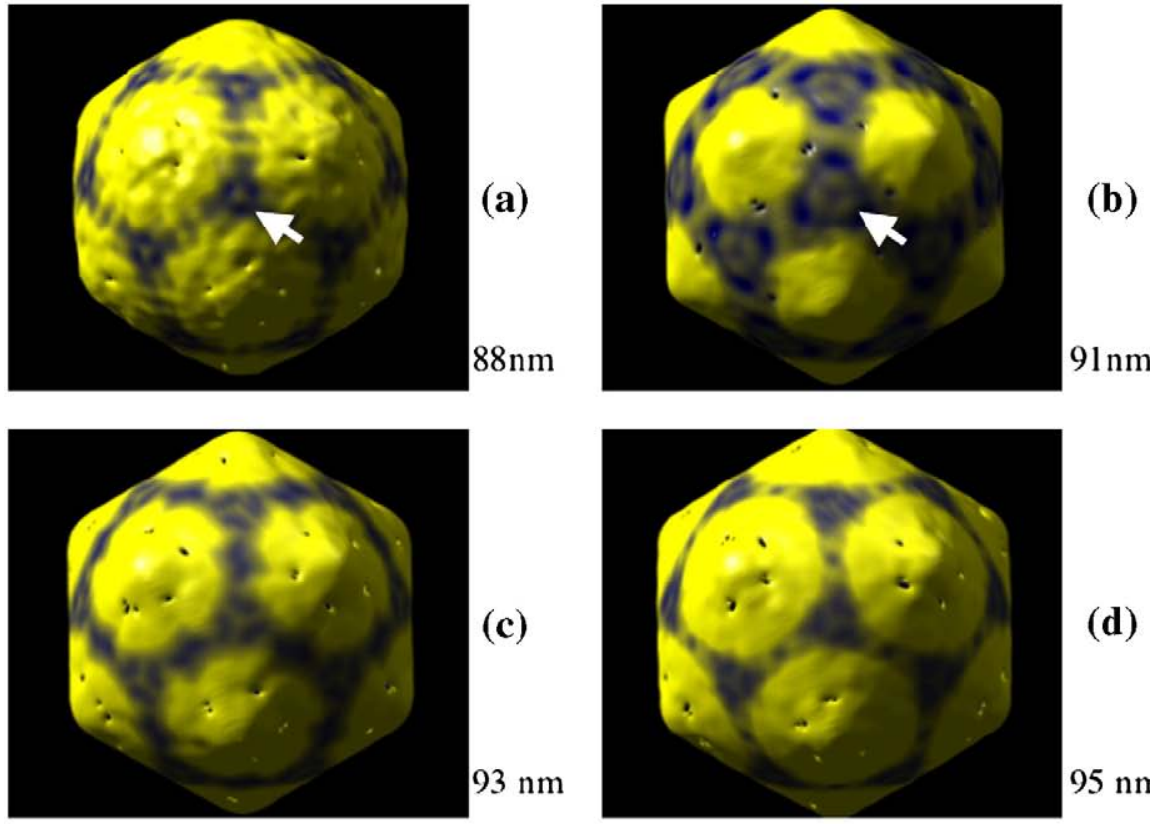

(c)
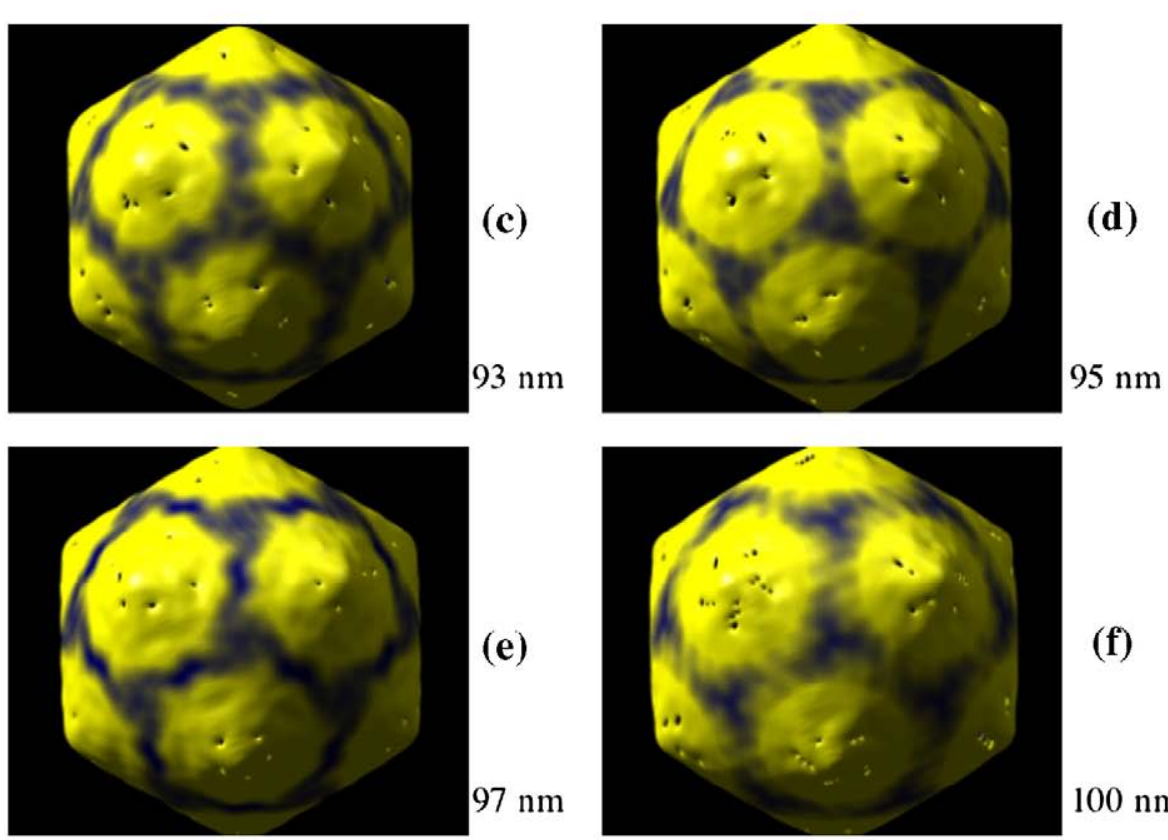

$93 \mathrm{~nm}$

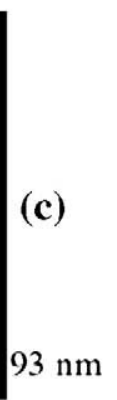

(d)

(e)

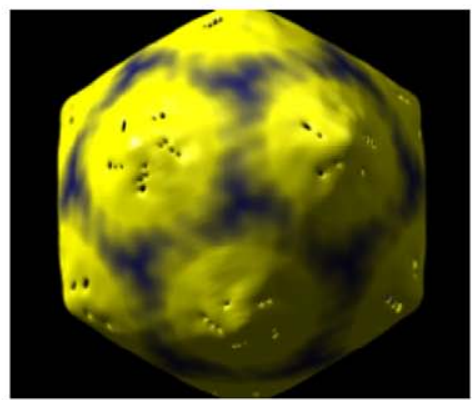

(f)

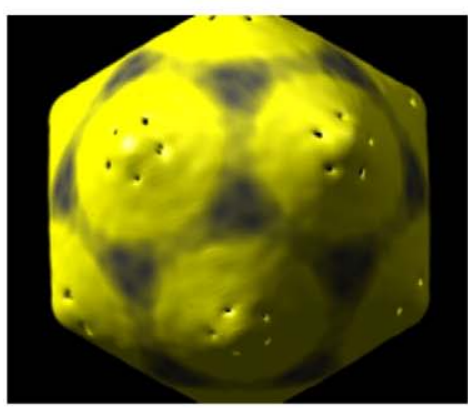

(g)

\section{Size classes}

$100 \mathrm{~nm}$

Figure 5. (a)-(g) A gallery of views down the 3-fold axis of all seven size classes, ranging in diameter from $88 \mathrm{~nm}$ to $103 \mathrm{~nm}$ in diameter. The surface has been colored radially to emphasize the low relief of the triangular faces. White arrows point to the different arrangement of density at the center of the triangular faces of two groups ((a) and (b)). Differences like this occur in all the groups. The variation in arrangement of the surface densities may reflect a different organization of the shell in different sized particles. The number of particle 3D volumes that went into each size class shown was: 3, 3, $13,18,17,21$, and 15 for (a)-(g), respectively. This is a reflection of the distribution of particle sizes shown in Figure 3.

\section{STEM measurements}

To determine the extent to which carboxysomes vary, STEM ${ }^{20}$ was used to make mass measurements of individual carboxysomes. The results in Figure 7 show that carboxysomes vary widely between 100 $\mathrm{MDa}$ and $350 \mathrm{MDa}$ with the majority populating the $190 \mathrm{MDa}-280 \mathrm{MDa}$ range. These results along with the cryo-images (Figure 1) and the histogram of their diameters (Figure 3) showing purified carboxysomes of varying sizes indicate that carboxysomes are not uniform in either size or mass.

\section{Discussion}

\section{Missing wedge in tomography}

The carboxysome is a large particle of variable size. It is difficult to determine its structure by 


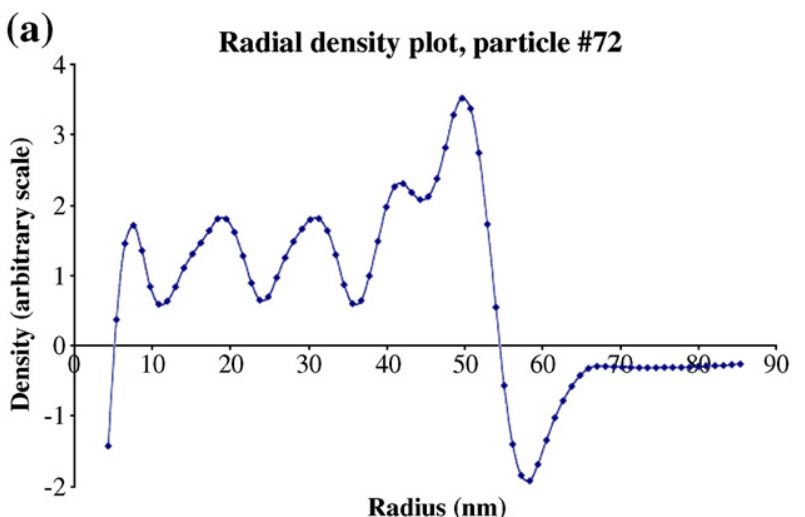

(b)

\section{Central slice of averaged particle $-100 \mathrm{~nm}$ class}

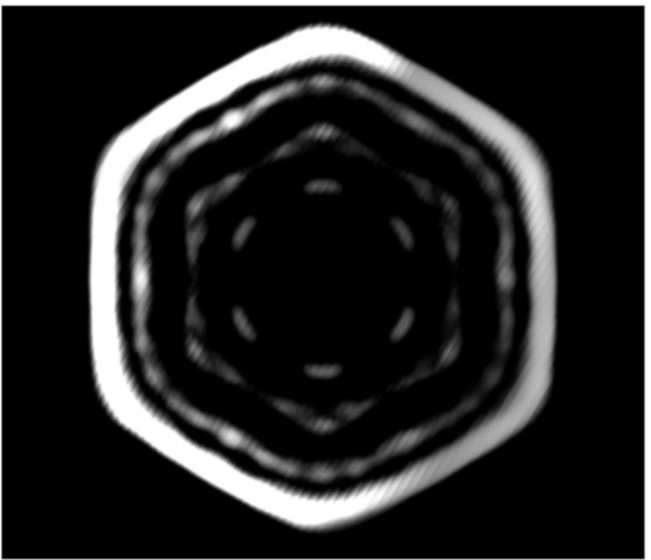

Figure 6. (a) A radial density distribution plot for one of the 3D particles that went into the average shown in Figure 4(a). The layered nature of the interior RuBisCO molecules is clear for each individual particle, though the exact azimuthal location turns out to be variable. (b) A projection of the central $12 \mathrm{~nm}$ thickness of the icosahedrally averaged particles of average size $100 \mathrm{~nm}$ (Figures 4(b) and 5(f)). The shell is of higher density because the interior is less regularly arranged and averaging decreases its density. However, the interior layers are spaced similar to the radial plot of the individual particles. The layer under the shell has the highest density, indicating that its average occupancy is higher than the layers closer to the center. The density threshold for this Figure had to be reduced to about half of what is used for Figure 5(f) in order to make the interior visible at all.

conventional single particle cryo-electron microscopy (EM) methods. Cryo-electron tomography is a technique that can give low resolution information but suffers from the problem of missing data due to limited tilt capability in a conventional electron microscope. If the particles are similar enough to average (at least into several groups if not one group) and randomly oriented, the averaging can alleviate this problem of missing data. Our methods for dealing with the missing wedge of data in Fourier space represent an attempt to deal with this problem in tomography, both in determining the relative orientation of extracted particles, each having this missing wedge, and in merging and averaging the $3 \mathrm{D}$ data from particles having these characteristics. Previous studies ${ }^{15,16}$ started with a search model that did not itself contain a missing wedge, or used alignment by translational, not rotational, correlation. ${ }^{21}$ When combined with the serious problem of size heterogeneity and incomplete prior knowledge of the symmetry, this is a difficult specimen to tackle by either single particle or tomographic methods. The methods described here can overcome much of the effect of the missing data, when objects in the tomographic reconstruction having different orientations can be averaged. When this is not possible, it may be necessary to minimize the missing data by other means, such as by dual axis tilting. ${ }^{22}$

\section{Merging and averaging heterogeneous size particles in tomograms}

Our strategy for dealing with the problem of heterogeneous particle population was to undertake multiple steps of classification. The first step allowed us to identify particles that have structural similarity with other particles. This eliminated particles with broken or incomplete shells, or completely empty particles, for example. The second step was to separate the data into particle size groups. The final distribution of size classes had seven groups, with average diameters of from $88 \mathrm{~nm}$ to $103 \mathrm{~nm}$, respectively. The distribution was not equal, and a majority of particles were in size classes that centered about 94-95 $\mathrm{nm}$ and 97-100 nm (Figure 3). The STEM measurements (Figure 7) show a broad distribution in carboxysome mass. This distribution in mass probably reflects the variable fraction of packing of RuBisCO molecules in carboxysomes of any size, in addition to the size variation itself.

\section{Symmetry determination}

Part of the confusion about the symmetry of the carboxysome was that it was not possible to find the orientation of the particles when the traditional "self common line" method ${ }^{23}$ was applied (A. M. P., unpublished results). In view of the structural results, there are several possible reasons for this failure. Prominent among these is the apparent lack of icosahedral symmetry in the internal RuBisCO, which comprises a large fraction of the mass. However, viruses may also have asymmetric internal mass similar to carboxysomes, or other nonicosahedral features, but do not show the same alignment problems using the standard icosahedral single particle reconstruction methods. We believe that the problem lies in the thin, flat triangular faces of the carboxysome shell. The shell of the carboxysome is shaped like a regular icosahedron without prominent "capsomeres". This means that in Fourier space, the power spectrum is largely confined to directions normal to the faces, and is quite weak in other directions. Central sections in Fourier space 


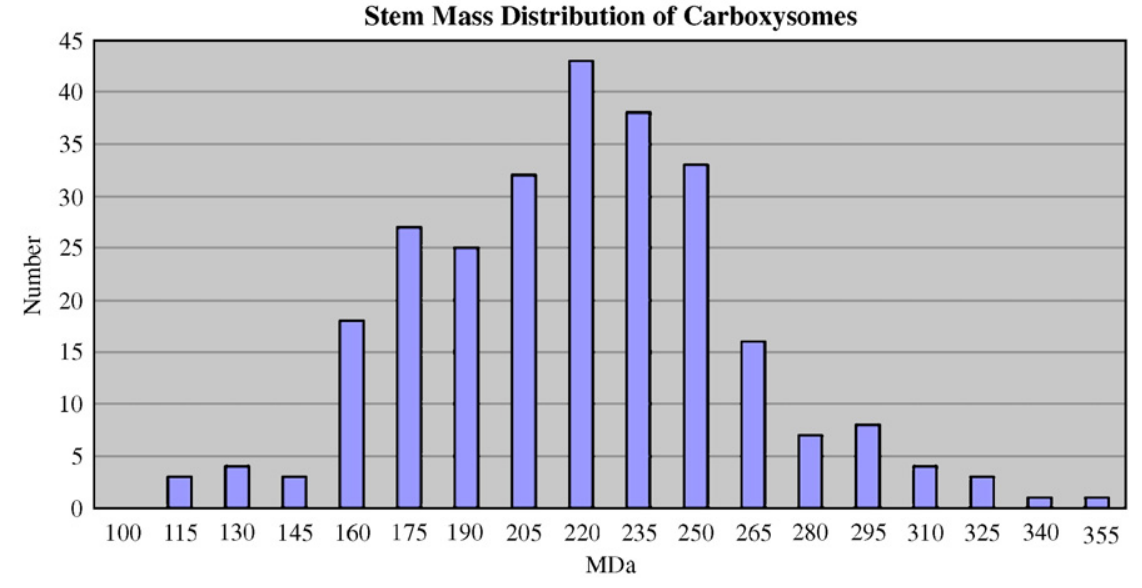

Figure 7. The mass distribution of purified $H$. neapolitanus carboxysomes determined by STEM mass measurements. The bins start at the labeled mass and are $15 \mathrm{MDa}$ wide. The $Y$ axis is the number of carboxysomes having the mass range indicated. It is interesting to note that the midpoint of this histogram (220 MDa) corresponds closely to a mass of protein forming a $100 \mathrm{~nm}$ diameter sphere with a density corresponding to a typical protein crystal. that do not contain these normals will have a weak icosahedrally symmetric signal. However, our 3D reconstruction from the tomograms was able to unambiguously indicate the icosahedral shape of the particle shell when properly aligned and averaged. Projection matching, ${ }^{24}$ which is useful when good starting models are available, should have fewer problems. In fact, a major result of this work is that we now have a series of reliable starting models of various sizes to begin to analyze the carboxysome by traditional single particle methods. Finally, the tomographic reconstruction reveals a tendency for the carboxysomes to have a preferred orientation, with their triangular faces toward the air-water interface. Preferred orientations are a potential source of problems in any single particle reconstruction project. Particles in this orientation tend to have a hexagonal outline in projection, which may have biased the search for other possible symmetry.

It should be noted that the subset of particles that were averaged does not include particles that may have been outliers in terms of their symmetry (nonicosahedral), size or shape, because these did not pass our original test of consistency with enough of the other particles in the tomogram to form their own classes. There are probably individual particles with other shapes and symmetry among the population. Also, these are particles isolated from bacteria, and the content and organization of their RuBisCO, for instance, could be different in vivo.

\section{Biological implications of observed structures and models}

The hexameric and sheet-like structure observed in the crystals of the $\mathrm{CcmK}$ proteins ${ }^{18}$ was proposed to be relevant to the structure of the shell. It is interesting and perhaps relevant that we observe density features on the surface of the carboxysome shell with spacing similar to that in the crystal (Figure 5(a)-(g)). In the crystal structure, one hexameric face has positive charges surrounding the center of the hexamer, forming a pore. ${ }^{18}$ However, in our carboxysomes, which contain up to eight other polypeptides, there are several other low-density features which may serve as "pores", some of which, near the vertices, appear considerably larger than those proposed from the homogeneous continuous sheets of $\mathrm{CcmK}$ proteins in the crystal structures.

Large and small carboxysomes were randomly distributed throughout the tomogram. This observation of variation in size and organization in an icosahedral particle is unusual, but not unprecedented. In mutant $\mathrm{P} 22^{25}$ and genetically engineered herpesvirus capsids, ${ }^{26}$ a variation in the composition or assembly leads to particles having different triangulation $(T)$ numbers. In carboxysomes, variations in the proportions or arrangement of the six to eight shell proteins could similarly produce carboxysomes of different sizes.

The different sized carboxysomes may have a different complement of protein components, or different diffusion and permeability characteristics for $\mathrm{CO}_{2}$ (which along with ribulose-1,5-bisphosphate is the productive substrate) and $\mathrm{O}_{2}$ (which is unproductive). Unlike a virus particle, where size variability is not compatible with packaging the required genome for viability, size variation of carboxysomes is non-lethal. In addition, carboxysomes are dynamic, and their appearance and RuBisCO content can change with physiological conditions. $^{11,12,2,27-29}$ Our concentric layer model of RuBisCO packing in biochemically isolated carboxysomes is the first that can account for both the reported "paracrystalline" packing which can fill the shell with equally spaced layers of molecules, as well as explain other observed arrangements that result in partially filled shells. The structures derived here may be useful in analyzing tomographic reconstructions of intact, live cyanobacteria to characterize the carboxysomes they contain in the native state.

\section{Materials and Methods}

\section{Vitrification of carboxysomes}

H. neapolitanus carboxysomes were first purified according to established methods. ${ }^{2,10}$ The specimen was then applied to 400 mesh copper EM grids, which had been coated with perforated carbon and glow discharged to make them hydrophilic. The perforated carbon consisted of 
a network of many $3-7 \mathrm{~mm}$ holes on the carbon film. ${ }^{30}$ Once applied to the EM grids, excess buffer was quickly wicked off with filter paper to suspend the carboxysomes in a thin film of buffer in holes within the carbon. The grids were then plunged into liquid ethane in a reservoir that was partially submerged in liquid nitrogen. ${ }^{31,32}$ After vitrification, the grids were stored under liquid nitrogen and later placed in the electron cryo-microscope for analysis.

\section{Cryo-electron tomography}

Images were recorded on a Gatan $4 \mathrm{k} \times 4 \mathrm{k}$ CCD camera on a JEM2010F electron microscope with an accelerating voltage of $200 \mathrm{kV}$ using the JAMES software package, ${ }^{33}$ which integrates the microscope, camera and database. Sample temperature was held at $102^{\circ} \mathrm{K}$ with a Gatan cryoholder. Single-axis tomographic imaging was performed manually at $20,000 \times$ magnification from $-58^{\circ}$ to $+70^{\circ}$ in $2^{\circ}$ increments under low-dose conditions. The total dose was kept under $6000 \mathrm{e}^{-} / \mathrm{nm}^{2}$. See Supplementary Data, Movie 1.

\section{Computer processing}

Tomographic reconstructions were carried out using the IMOD package. ${ }^{34}$ Registration of the tomographic images to each other was accomplished by recording the positions of about seven ice contamination masses and the centers of three carboxysomes, which could be followed through most of the tilt series. These were used as fiducials in the IMOD software suite to refine the tilt angles, axis and magnification of each image. In contrast to the normal procedures with this software, where gold particles are used, the "fiducials" in this case had to be recorded manually in each image, because automated tracking was not feasible.

Subsequent processing, including alignment, symmetrization, averaging, calculating $1 \mathrm{D}$ radial plots from the $3 \mathrm{D}$ volumes, etc. of the $3 \mathrm{D}$ particle volumes, was done using available EMAN (and EMAN2) routines, ${ }^{24}$ or by python scripts that make use of EMAN (and EMAN2) procedures to implement the improvements in orientation searching, averaging and merging that are discussed in the text. Visualization was done by $3 \mathrm{dmod}$ (part of the IMOD package) and by Chimera. ${ }^{35}$

\section{STEM mass determination}

Briefly, carboxysomes in buffer were applied to $2.3 \mathrm{~mm}$ titanium grids coated with a thin carbon support film on a layer of holey carbon film. The specimen was freeze-dried according to the methods established by the Brookhaven National Laboratory. The freeze-dried carboxysomes were then placed into the STEM and maintained at $-150{ }^{\circ} \mathrm{C}$. The specimen was then scanned with a probe from the microscope operating at $40 \mathrm{keV}$ and with a probe size of $0.25 \mathrm{~nm}$. Rasters of $512 \times 512$ areas of the specimen were recorded. Elastically scattered electrons were digitally recorded using both a large and small angle detector. These images were analyzed and compared to standards to determine mass. ${ }^{20}$

\section{Acknowledgements}

This research was supported by the grant P41RR02250. Dr Gordon Cannon at the University of Southern Mississippi, Hattiesburg, provided the carboxysomes used for STEM mass measurements; Dr Martha Simon at the Brookhaven National Laboratory, a NIH supported resource center (NIH 5 P41EB2181) conducted STEM mass measurements of carboxysomes. We also thank Michael Marsh and Drs Christopher Booth and Steven Ludtke for helpful discussions.

\section{Supplementary Data}

Supplementary data associated with this article can be found, in the online version, at doi:10.1016/ j.jmb.2006.09.024

\section{References}

1. Shively, J. M., Ball, F., Brown, D. H. \& Saunders, R. E. (1973). Functional organelles in prokaryotes: polyhedral inclusions (carboxysomes) of Thiobacillus neapolitanus. Science, 182, 584-586.

2. Cannon, G. C. \& Shively, J. M. (1983). Characterization of a homogenous preparation of carboxysomes from Thiobacillus neapolitanus. Arch. Microbiol. 134, 52-59.

3. Shively, J. M. (1974). Inclusion bodies of prokaryotes. Annu. Rev. Microbiol. 28, 167-187.

4. Codd, G. A. \& Marsden, W. J. N. (1984). The carboxysomes (polyhedral bodies) of autotrophic prokaryotes. Biol. Rev. 59, 389-422.

5. Shively, J. M. \& English, R. S. (1991). The carboxysome, a prokaryotic organelle: a mini review. Can. J. Bot. 69, 957-962.

6. Cannon, G. C., Bradburne, C. E., Aldrich, H. C., Baker, S. H., Heinhorst, S. \& Shively, J. M. (2001). Microcompartments in prokaryotes: carboxysomes and related polyhedra. Appl. Environ. Microbiol. 67, 5351-5361.

7. Heinhorst, S., Cannon, G. \& Shively, J. M. (2006). Carboxysomes and carboxysome-like inclusions. In Microbiology Monographs (Shively, J. M., ed), vol. 2. Complex intracellular structures in prokaryotes, pp. 141-166. Springer, Berlin, Heidelberg, New York.

8. Shively, J. M., Ball, F. L. \& Kline, B. W. (1973). Electron microscopy of the carboxysomes (polyhedral bodies) of Thiobacillus neapolitanus. J. Bacteriol. 116, 1405-1411.

9. Holthuijzen, Y., van Breemen, J. F. L., Konig, W. N. \& van Bruggen, E. F. J. (1986). Electron microscopic studies of carboxysomes of Thiobacillus neapolitanus. Arch. Microbiol. 144, 258-262.

10. So, A. K., Espie, G. S., Williams, E. B., Shively, J. M., Heinhorst, S. \& Cannon, G. C. (2004). A novel evolutionary lineage of carbonic anhydrase (epsilon class) is a component of the carboxysome shell. J. Bacteriol. 186, 623-630.

11. Orus, M. I., Rodriguez-Buey, M. L., Marco, E. \& Fernandez-Valiente, E. (2001). Changes in carboxysome structure and grouping and in photosynthetic affinity for inorganic carbon in Anabaena strain PCC 7119 (Cyanophyta) in response to modification of CO2 and Na+ supply. Plant. Cell. Physiol. 42, 46-53.

12. Woodger, F. J., Badger, M. R. \& Price, G. D. (2003). Inorganic carbon limitation induces transcripts encoding components of the $\mathrm{CO}(2)$-concentrating mechanism in Synechococcus sp. PCC7942 through a redoxindependent pathway. Plant Physiol. 133, 2069-2080.

13. Peters, K. R. (1974). Characterization of a phage-like particle from cells of Nitrobacter. II. Structure and size (author's transl). Arch. Microbiol. 97, 129-140. 
14. Kaneko, Y., Danev, R., Nagayama, K. \& Nakamoto, H. (2006). Intact carboxysomes in a cyanobacterial cell visualized by hilbert differential contrast transmission electron microscopy. J. Bacteriol. 188, 805-808.

15. Walz, J., Typke, D., Nitsch, M., Koster, A. J., Hegerl, R. \& Baumeister, W. (1997). Electron Tomography of Single Ice-Embedded Macromolecules: Three-Dimensional Alignment and Classification. J. Struct. Biol. 120, 387-395.

16. Forster, F., Medalia, O., Zauberman, N., Baumeister, W. \& Fass, D. (2005). Retrovirus envelope protein complex structure in situ studied by cryoelectron tomography. Proc. Natl Acad. Sci. USA, 102, 4729-4734.

17. Schmid, M. F. (2003). Cross-correlation and merging of crystallographic reflections derived from cryo-electron micrographs of 3D crystals: application to the Limulus acrosomal bundle. J. Struct. Biol. 144, 195-208.

18. Kerfeld, C. A., Sawaya, M. R., Tanaka, S., Nguyen, C. V., Phillips, M., Beeby, M. \& Yeates, T. O. (2005). Protein structures forming the shell of primitive bacterial organelles. Science, 309, 936-938.

19. Sawaya, M. R. Cannon, G. C., Heinhorst, S., Tanaka, S., Williams, E. B., Yeates, T. O. \& Kerfeld, C. A. (2006). The structure of beta-arbonic anhydrase from the carboxysomal shell reveals a distinct subclass with one active site for the price of two. J. Biol. Chem. 281, 7546-7555.

20. Wall, J. S., Hainfeld, J. F. \& Simon, M. N. (1998). Scanning transmission electron microscopy of nuclear structures. Methods Cell Biol. 53, 139-164.

21. Nicastro, D., McIntosh, J. R. \& Baumeister, W. (2005). 3D structure of eukaryotic flagella in a quiescent state revealed by cryo-electron tomography. Proc. Natl Acad. Sci. USA, 102, 15889-15894.

22. Iancu, C. V., Wright, E. R., Benjamin, J., Tivol, W. F., Dias, D. P., Murphy, G. E. et al. (2005). A "flip-flop" rotation stage for routine dual-axis electron cryotomography. J. Struct. Biol. 151, 288-297.

23. Crowther, R. A., Henderson, R. \& Smith, J. M. (1996). MRC image processing programs. J. Struct. Biol. 116, 9-16.

24. Ludtke, S. J. (1999). EMAN: semiautomated software for high-resolution single-particle reconstructions. J. Struct. Biol. 128, 82-97.
25. Thuman-Commike, P. A., Greene, B., Malinski, J. A., King, J. \& Chiu, W. (1998). Role of the scaffolding protein in P22 procapsid size determination suggested by $\mathrm{T}=4$ and $\mathrm{T}=7$ procapsid structures. Biophys. J. 74, 559-568.

26. Saad, A., Zhou, Z. H., Jakana, J., Chiu, W. \& Rixon, F. J. (1999). Roles of triplex and scaffolding proteins in herpes simplex virus type 1 capsid formation suggested by structures of recombinant particles. J .Virol. 73, 6821-6830.

27. Turpin, D., Miller, A. \& Canvin, D. (1984). Carboxysome content Synechococcus leopoliensis (Cyanophyta) in response to inorganic carbon. J. Phycol. 20, 249-253.

28. McKay, R., Gibbs, S. \& Espie, G. (1993). Effect of dissolved inorganic carbon on the expression of carboxysomes, localization of RuBisCO and mode of inorganic carbon transport in cells of the cyanobacterium Synechococcus. Arch. Microbiol. 159, 21-29.

29. Beudeker, R., Cannon, G., Keunen, J. \& Shively, J. M. (1980). Relations between D-ribulose-1,5-bisphosphate carboxylase, carboxysomes, and CO2 fixing capacity in the obligate chemolithotroph Thiobacillus neapolitanus grown under different limitations in the chemostat. Arch. Microbiol. 124, 185-189.

30. Toyoshima, C. (1989). On the use of holey grids in electron crystallography. Ultramicroscopy, 30, 439-444.

31. Adrian, M., Dubochet, J., Lepault, J. \& McDowall, A. W. (1984). Cryo-electron microscopy of viruses. Nature, 308, 32-36.

32. Lepault, J., Booy, F. P. \& Dubochet, J. (1983). Electron microscopy of frozen biological suspensions. J. Microsc. 129, 89-102.

33. Booth, C. R., Jiang, W., Baker, M. L., Zhou, Z. H., Ludtke, S. J. \& Chiu, W. (2004). A 9 angstroms single particle reconstruction from CCD captured images on a $200 \mathrm{kV}$ electron cryomicroscope. J. Struct. Biol. 147, 116-127.

34. Kremer, J. R., Mastronarde, D. N. \& McIntosh, J. R. (1996). Computer visualization of three-dimensional image data using IMOD. J. Struct. Biol. 116, 71-76.

35. Pettersen, E. F., Goddard, T. D., Huang, C. C., Couch, G. S., Greenblatt, D. M., Meng, E. C. \& Ferrin, T. E. (2004). UCSF Chimera-a visualization system for exploratory research and analysis. J. Comput. Chem. 25, 1605-1612. 